\title{
Uncertainty Theories Based Iris Recognition System
}

\author{
Majd BELLAAJ, Imen KHANFIR KALLEL and Dorra SELLAMI \\ CEM Lab, National Engineering School of Sfax, University of Sfax, 3038 Sfax, Tunisia \\ (iTi) Department, IMT Atlantique, France
}

Received 27th Jul 2017; accepted 11th Jan 2018

\begin{abstract}
The performance and robustness of the iris-based recognition systems still suffer from imperfection in the biometric information. This paper makes an attempt to address these imperfections and deals with important problem for real system. We proposed a new method for iris recognition system based on uncertainty theories to treat imperfection iris feature. Several factors cause different types of degradation in iris data such as the poor quality of the acquired pictures, the partial occlusion of the iris region due to light spots, or lenses, eyeglasses, hair or eyelids, and adverse illumination and/or contrast. All of these factors are open problems in the field of iris recognition and affect the performance of iris segmentation, its feature extraction or decision making process, and appear as imperfections in the extracted iris feature. The aim of our experiments is to model the variability and ambiguity in the iris data with the uncertainty theories. This paper illustrates the importance of the use of this theory for modeling or/and treating encountered imperfections. Several comparative experiments are conducted on two subsets of the CASIA-V4 iris image database namely Interval and Synthetic. Compared to a typical iris recognition system relying on the uncertainty theories, experimental results show that our proposed model improves the iris recognition system in terms of Equal Error Rates (EER), Area Under the receiver operating characteristics Curve (AUC) and Accuracy Recognition Rate (ARR) statistics.

Key Words: Iris Recognition System, Iris Feature Imperfections, Probability Theory, Possibility Theory.
\end{abstract}

\section{$1 \quad$ Introduction}

Iris recognition system [1] is the process of recognizing a person by analyzing the random pattern of his iris. The iris is the colored part of the eye and is around the pupil of every human being. In fact, it is a muscle within the eye that regulates the size of the pupil, controlling the amount of light that enters the eye. This biometric trait has a distinct texture containing randomly distributed and irregularly shaped microstructures, unique and informative texture patterns. The current research of the human iris could be classified into three main categories namely: the iris segmentation [2],[3], feature extraction and iris signature [4],[5], eyelid or eyelash detection [6], and compensation of eye rotation and iris texture deformation [7].

The efficiency and robustness of the human iris are greatly relying on the quality of the captured biometric sample. Recently, a great deal of research [3],[8],[9] was applied on non ideal iris images. There are many situations where the iris texture is damaged, e.g. in the case of lenses, eyeglasses, hair, motion blur, non-linear, deformed, ageing or adverse illumination and compression. All of these affect the iris template (the extracted iris feature). Therefore, it is imperative to first analyze the iris data and look for correcting the type of imperfections induced. Such imperfections can be classified into two main groups: imprecision and uncertainty [10]. Depending on the imperfection type, the state-of-theart offers several tools proposed to deal with uncertain information such as, probability theory, fuzzy set theory [11],

Correspondence to: < majd.bellaj@gmail.com >

Recommended for acceptance by $<$ Anjan Dutta and Carles Sánchez $>$

DOI : https://doi.org/10.5565/rev/elcvia.1131

ELCVIA ISSN: 1577-5097

Published by Computer Vision Center / Universitat Autònoma de Barcelona, Barcelona, Spain 
evidence theory [12] and possibility theory [13]. Given the diversity of these uncertainty theories, we aim to choose the best method that can be adapted to our context. The specificity of the iris biometric data suggests a model able to deal with both ambiguity and variability. The probability theory was the first uncertainty theory that handles imperfect information but seems to be inappropriate due to its limitation in managing uncertainty without being able to handle imprecision. Furthermore, the fuzzy set theory is well suited for modeling imprecision and more specifically, ambiguity but it is unable to handle uncertainty. Fortunately, the evidence theory is able to model some types of uncertain or imprecise information. Such formalism is provided by the possibility theory.

The possibility theory seems to fit best our proposed iris recognition system. Indeed, this uncertainty theory is a natural and flexible tool for representing imperfect information that is uncertain, more precisely variability and imprecise, more precisely ambiguity. Zadeh [14] was the first to introduce this theory and, subsequently, it was improved by several other authors, like Dubois and Prade [13], Cooman and Aeyels [15] among others. This theory has developed rapidly in recent years and has been widely used in various fields such as, diagnosis [16], obstacle avoidance systems and electronic travel aids (ETA) [17], and biometrics [18],[19].

In the possibility theory, the possibility distribution [15] is an essential concept, that can be derived from the collected data. This theory is related to other theories [20], such as, the probability theory, the fuzzy set theory and the belief function theory. The transformations (probabilistic-possibilistic, membership function- possibilistic or basic belief assignment-possibilistic) applied to these rules are often accompanied with a loss of information, i.e., an increase of imperfection (imprecision and/or uncertainty). Thus, a special attention should be paid. In our approach, we chose the probabilistic-possibilistic transformation, because this technique perfectly manipulates most of the imperfections related to our biometric data without loss of relevant information. Besides, these transformations are useful especially when dealing with uncertain and imprecise information.

Many techniques have been developed to obtain a possibility distribution based on the probability distribution [20][23]. They can be classified into two categories of probability-possibility transformations; one is based on the precise probability value and the other on the probability interval. The choice of the method and the class of probabilitypossibility transformation, meeting the requirements of our iris modality, is a crucial step in our work. Variability and ambiguity, present in our iris data, make it difficult to get an accurate probability value. Thus, the obtained possibility distribution may not be satisfactory. To overcome this limitation, the probability confidence intervals offer an accurate tool for modeling such imperfections. Accordingly, the probabilistic-possibilistic transformation of Campos and Huete [24], relying on the probability intervals, is more able to cope with collected biometric data imperfections.

\section{Motivation and contribution}

An iris recognition system can be efficient if it is applied to a specific database but gives unsatisfactory results when applied to another database. By modeling the imperfection information contained in the extracted iris feature with the uncertainty theories, we hope to have a generic and performing iris system despite the errors that may occur during the acquisition or segmentation of the iris and the used normalization or extraction method. The main goal of our work was to build a robust iris recognition system to solve the new difficulties faced in this area. We are not trying to build a new method of localization of iris or an efficient extraction method of relevant information because, from the literature, we have seen that the performance of these methods varies according to the quality of the iris image. For this reason, we have chosen to treat imperfect information. The results show that our proposed system based on the uncertainty theories improves robustness and reliability in terms of identification and the proposed iris feature imperfection modeling approach was very effective in treating the imperfections found in the iris signature. Our contribution has consequently improved the performance of the typical iris recognition system [28].

\section{Brief Method}

In this paper, we propose an improved approach essentially based on iris feature imperfection modeling process. This modeling step is divided into four stages: In the first stage, the extracted feature is transformed into normalized measurements by Z-score and Min-Max normalization methods [25]. In the second step, the normalized feature is transformed into histogram probability distribution based on histogram parameters [26]. In the third step, the histogram probability distribution is transformed into interval probability distribution using Goodman formalism [27]. Finally, in the fourth step, the interval probability distribution is transformed into possibility distribution relying on Campos and Huete approach [24]. For the matching process, a similarity measure based on Manhattan distance is applied. Different experiments are performed on two subsets of CASIA-V4 iris image database to assess the proposed system. The purpose of our study is to apply the uncertainty theories for overcoming iris-data biometric system imperfections. 


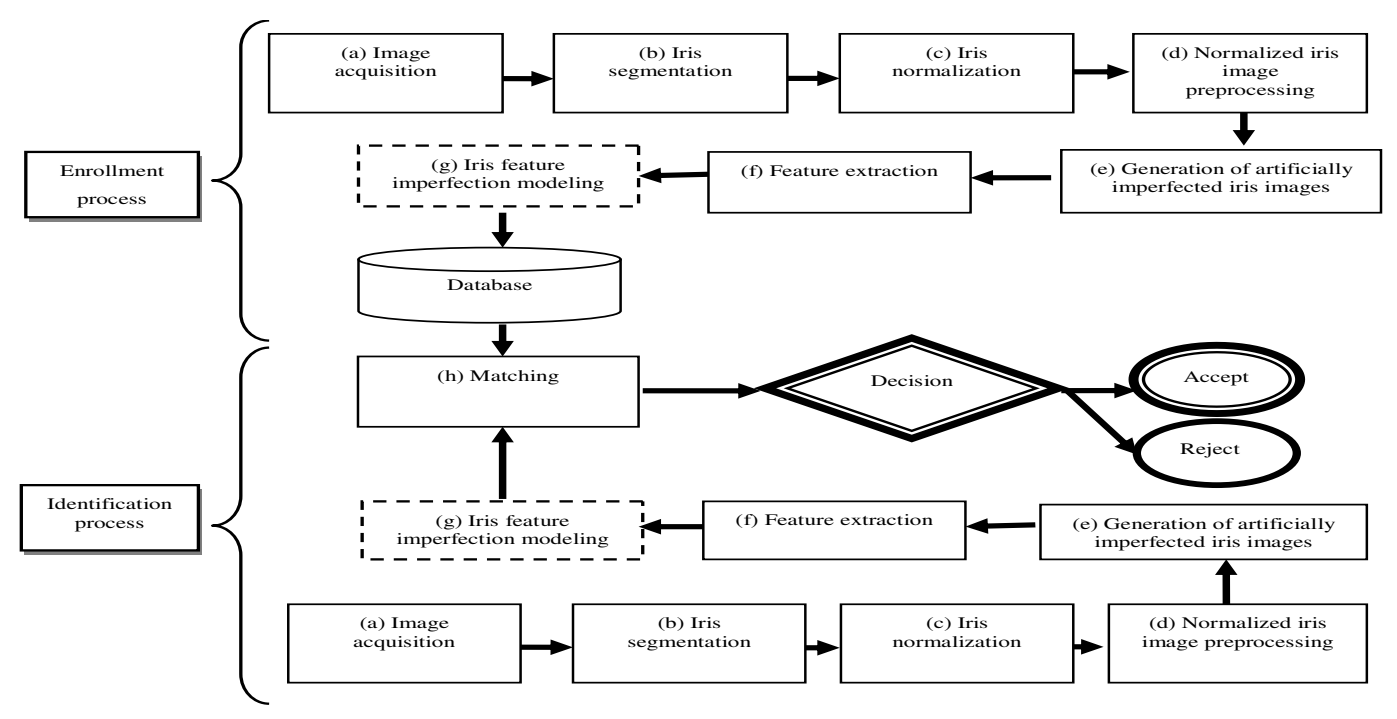

Fig 1: Flow of the proposed iris recognition system: (a) image acquisition; (b) iris segmentation; (c) iris normalization; (d) normalized iris image preprocessing; (e) generation of artificially imperfected iris images; (f) feature extraction; (g) iris feature imperfection modeling; (h) matching.

\section{Brief results}

In order to have a baseline for the comparison of the achieved results of the proposed method, we compared our iris recognition system to a typical one [28]. This biometric system consists of almost the same steps and methods as our proposed system, except for the iris feature imperfection modeling step (Fig. 1 (g)) which was omitted and the decision stage (Fig. 1 (f)) is achieved relying on the normalized Manhattan distance. In order to compare the proposed iris recognition system and that of the typical system [28], we extracted a subset of image samples from the CASIA-V4Interval iris image database, including 700 left eye images of 100 subjects, having therefore 7 images per eye, whereas, for the CASIA-V4-Synthetic iris image database, we used all images of this database which contains 1000 subjects and 10,000 iris images. In our experiments, the EER, AUC and finally the ARR statistics were computed and used for the performance assessment of both proposed and typical [28] systems. In our experiments, it is proposed to give the corresponding rate only to the testing phase. A first experiment to assess performance of the proposed iris system was conducted with a subset of right eye image samples from the CASIA-V4-Interval iris image database.

The AUC, EER and ARR were used to assess the overall performance of both systems. The results of these assessments are shown also in Table 1. These outcomes confirm the improved performance yielded by the proposed system compared to the typical one [28] and indicating the feasibility of an iris recognition using a modeling iris feature step based on uncertainty theories. Following the encouraging results obtained in the first experiment using the step of modeling imperfections iris feature and applied on a CASIA-V4-Interval iris image database, additional experiments were also conducted on CASIA-V4-Synthetic iris image databases to assess the performance of our proposed iris system. Tables 2 give the values in terms of AUC, EER, and ARR for this database. The results obtained in this table demonstrate the recognition efficiency of the proposed biometric system. Table 3 shows the comparison between our proposed method and others methods [29]-[31] tested with samples iris images from CASIA-V4-Interval database. Observing the values on this table, we can see that the AUC and ARR of the proposed method significantly exceed the AUC and ARR of other methods. It is also noticed that the lower EER value are provided by the proposed method over the others. Therefore, we can assume that our method perfectly minimizes the recognition errors.

\section{Conclusions}

In this paper, we proposed a robust method for iris recognition system based on the uncertainty theories. The experimental results on two subsets of CASIA-V4 iris image database namely: Synthetic and Interval, show that the identification performance of the proposed system is improved, compared to other typical iris recognition system. It proves the robustness of our approach dealing with some imperfections related to the iris biometric data. It should be noted that in the present work, our aim was to improve the performance of a recognition system regardless the use of ideal and/or non-ideal iris images from the database, or the imperfections that appear in the extracted iris feature. 


\begin{tabular}{ccc}
\hline $\begin{array}{c}\text { CASIA-V4-Interval } \\
\text { (100 subjects, 700 images) }\end{array}$ & $\begin{array}{c}\text { Typical system } \\
\text { (without proposed } \\
\text { approach) }\end{array}$ & $\begin{array}{c}\text { Proposed system } \\
\text { (with proposed approach) }\end{array}$ \\
\hline AUC & 0.8647 & 0.9999 \\
EER(\%) & 21.3 & 0.06 \\
ARR $(\%)$ & 78.69 & 99.93 \\
\hline
\end{tabular}

Table 1: AUC, EER and ARR obtained for both systems on a subset of left eye image samples from CASIA-V4Interval iris image database.

\begin{tabular}{cc}
\hline $\begin{array}{c}\text { CASIA-Synthetic } \\
(1000 \text { subjects, } \\
(10000 \text { images })\end{array}$ & $\begin{array}{c}\text { Proposed system } \\
\text { (with proposed approach) }\end{array}$ \\
\hline AUC & 0.9999 \\
EER $(\%)$ & 0.14 \\
ARR $(\%)$ & 99.86 \\
\hline
\end{tabular}

Table 2: AUC, EER and ARR obtained for the proposed system on CASIA-V4-Synthetic iris image database.

\begin{tabular}{cccccc}
\hline $\begin{array}{c}\text { CASIA V4- } \\
\text { Interval }\end{array}$ & $\begin{array}{c}\text { Daugman method [31] } \\
(2015,500 \text { images })\end{array}$ & $\begin{array}{c}\text { Mira et al method } \\
{[31](500 \text { images })}\end{array}$ & $\begin{array}{c}\text { Saminathan et al method } \\
{[32](2015)}\end{array}$ & $\begin{array}{c}\text { Rathgeb et al. } \\
\text { Method [33] (2016) }\end{array}$ & $\begin{array}{c}\text { Proposed method } \\
(700 \text { images })\end{array}$ \\
\hline AUC & 0.9976 & 0.9975 & - & - & 0.9999 \\
EER(\%) & 1.05 & 1.12 & - & 0.98 & 0.06 \\
ARR(\%) & 98.95 & 98.88 & 98.5 & - & 99.93 \\
\hline
\end{tabular}

Table 3: Comparison between our proposed method and others methods

\section{References}

[1] J. G. Daugman, "Biometric Personal Identification System Based on Iris Analysis", United States Pattent, no.5291560, 1994

[2] S.Thainimit, C. Sreecholpech, V. Areekul and C. H. Chu, "Robust Iris Segmentation Based on Local Image Gradient Properties," IEICE Trans. Inf. \& Syst., vol. E94-D, no. 2, pp. 349-356, Feb. 2011.

[3] P. Li, X. Liua, L. Xiaoa, and Q. Songa, "Robust and Accurate Iris Segmentation in Very Noisy Iris Images," Image and Vision Computing, vol.28, no.2, pp. 246-253, 2010.

[4] J. Sun, L. Zhou, Z. Lu and T. Nie," Iris Recognition Based on Local Gabor Orientation Feature Extraction," IEICE Trans. Inf. \& Syst., vol. E98-D, no. 8, pp. 1604-1608, Aug. 2015.

[5] S. Noh, K. Bae, K. R. Park and J. Kim," A New Iris Recognition Method Using Independent Component Analysis," IEICE Trans. Inf. \& Syst., vol. E88-D, no. 11, pp. 2573-2581, Nov.2005.

[6] T. Mina and R. Parka, "Eyelid and Eyelash Detection Method in the Normalized Iris Image Using the Parabolic Hough model and Otsu's Thresholding Method," Pattern Recognition Letters, vol. 30, no. 12, pp. 1138-1143, 2009.

[7] H. Takano, H. Kobayashi, and K. Nakamura, "Rotation Invariant Iris Recognition Method Adaptive to Ambient Lighting Variation," IEICE Trans. Inf. \& Syst., vol.E90-D, no. 6, pp. 955-962, Jun. 2007.

[8] P. Grother, J. R. Matey, E. Tabassi, G. W. Quinn, M. Chumakov, "IREX VI: Temporal Stability of Iris Recognition Accuracy (Iris Ageing)," NIST Interagency Report 7948, July. 2013.

[9] S. H. Moi, H. Asmuni, R. Hassan, R. M. Othman," Multimodal Biometrics:Weighted Score Level Fusion Based on Non-ideal Iris and Face Images," Expert Systems with Applications, vol. 41, no. $11,2014$.

[10] A.Motro and P. Smets, eds., Uncertainty Management in Information Systems, Springer Science and Business Media New York, 1997.

[11] L. A. Zadeh, "Fuzzy Sets," Information and Control," vol. 8, pp. 338-353, 1965.

[12] G. A. Shafer, ed Mathematical Theory of Evidence, Princeton Univ. Press, 1976

[13] D. Dubois and H. Prade, eds., Possibility Theory: An Approach to Computerized Processing of Uncertainty, Plenum Press New York, 1988.

[13] D. Dubois and H. Prade, eds., Possibility Theory: An Approach to Computerized Processing of Uncertainty, Plenum Press

[14] L. A. Zadeh, "Fuzzy Sets as a Basis for a Theory of Possibility," Fuzzy Sets and Systems, vol. 100, no. 1, pp. 9-43, 1999.
[15] G. Cooman and D. Aeyels, "A Random Set Description of a Possibility Measure and its Natural Extension," IEEE Trans. on Syst, Man and Cybernetics, vol. 30, no. 2, pp. $124-130,2000$.

[15] G. Cooman and D. Aeyels, "A Random Set Description of a Possibility Measure and its Natural Extension," IEEE Trans. on Syst, Man and Cybernetics, vol. 30, no. 2, pp. 124-130, 2000.
[16] D. Dubois, D. Cayrac and H. Prade, "Handling Uncertainty with Possibility Theory and Fuzzy Sets in a Satellite Fault Diagnosis Application," Proc. 6th Int. Conf. on Information Processing and . Dubois, D. Cayrac and H. Prade, "Handling Uncertainty with Possibility Theory and Fuzzy
Management of Uncertainty in Knowledge Based Systems, vol. 4, no. 3, pp. 251-269, 1996.

[17] J. Frikha, D. Sellami, and I. Khanfir Kallel, "Indoor/outdoor navigation system based on possibilistic traversable area segmentation for visually impaired people," ELCVIA Electron. Lett. Comput. Vis. Image Anal., vol. 15, no. 1, p. 60, Aug. 2016

[18] Bellaaj M.,. Masmoudi D. S and Kallel I. K.,"Possibilistic Modeling of Iris System for High Recognition Reliability", IJCSNS International Journal of Computer Science and Network Security, Thomson Reuters Publishing, Volume Number: Vol.16, No.7, pp.34-47, July. 2016

[19] M. Bellaaj, J. F. Elleuch, D. S. Masmoudi and I. K. Kallel, "An Improved Iris Recognition System Based on Possibilistic Modeling," Proc. 13th Int. Conf. on Advanced in Mobile Computing and Multimedia (MoMM), Brussels, Belgium, pp. 26-32, Dec. 2015.

[20] I.Jenhani, "From Possibilistic Similarity Measures to Possibilistic Decision Trees", thesis for obtaining the PhD from the University of Artois, French, 2010.

[21] D.Dubois, H.Prade and S.Sandri, "On Possibility/probability Transformations," in Theory and Decision Library, eds. W. Leinfellner, G. Eberlein, R. Lowen, vol. 12, pp. $103-112,1993$.

[22] M. Masson and T. Denoeux, "Inferring a Possibility Distribution from Empirical Data," Fuzzy Sets and Systems, vol. 157, no. 3, pp. 319-340, 2006.

[23] D.Dubois and H. Prade, "Représentations Formelles de l'Incertain et de l'Imprécision," in Concepts et Méthodes pour l'Aide à la Décision, ed. Hermes-Lavoisier, vol. 1, pp. 111-165, Lavoisier S.A.S., 2006.

[24] L. M. Campos and J. F. Huete, "Measurement of Possibility Distributions," Int. J. General Systems, vol. 30, no. 3, pp. 309-346, 2001.

[25] F. Alsade, N. zaman, M. Z. Dawood and S. H. A. Musavi, "Effectiveness of Score Normalisation in Multimodal Biometric Fusion," Journal of ICT, vol. 3, no. 1, pp. 29-35, 2009.

[26] M. S. Mouchaweh and P. Billaudel, "Influence of the Choice of Histogram Parameters at Fuzzy Pattern Matching Performance", WSEAS Transactions on Systems, vol. 1, no. 2, pp. 260-266, 2002.

[27] L. A. Goodman, "On Simultaneous Confidence Intervals for Multinomial Proportions," Technometrics, vol. 7, no. 2, pp. 247-254, 1965.

[28] I. Khanfir, "Contribution à l'Identification d'Individus par l'Iris," thesis for obtaining the PhD in Electrical Engineering from the National Engineering School of Sfax, Tunisia, 2009.

[29] W. L. May and W.D. Johnson, "A SAS Macro for Constructing Simultaneous Confidence Intervals for Multinomial Proportions", Computer Methods and Programs in Biomedecine, vol. 53, no. 3 , pp. 153-162, July. 1997.

[30] C. P Sison and J. Glaz, "Simultaneous Confidence Intervals and Sample Size Determination for Multinomial Proportions", Journal of the American Statistical Association, vol. 90, no. 429, pp.

[31] Mira Jr. J., Neto H. V., Nevesand E. B, Schneider F. K., "Biometric-oriented Iris Identification Based on Mathematical Morphology", Journal of Signal Processing Systems, vol. 80, no. 2, pp. 181195, Aug. 2015.

[32] Saminathan, K., Chakravarthy, T., and M. Chithra Devi. “Iris Recognition Based On Kernels Of Support Vector Machine”, Ictact Journal on Soft Computing, Volume: 05, Issue: 02, pp, 889-895, 2015.

[33] Rathgeb C., Uhl A., Wild P., and Hofbauer H., "Design Decisions for an Iris Recognition SDK," in Handbook of Iris Recognition, K. W. Bowyer and M. J. Burge, Eds. Springer London, pp. 359$396,2016$. 